\title{
Estrogen receptor (ESR1) mutation in bone metastases from breast cancer
}

\author{
Stephan Bartels, Matthias Christgen, Angelina Luft, Sascha Persing, Kai Jödecke, \\ Ulrich Lehmann and Hans Kreipe \\ Institute of Pathology, Hannover Medical School, Hannover, Germany
}

\begin{abstract}
Activating mutations of estrogen receptor $\alpha$ gene (ESR1) in breast cancer can cause endocrine resistance of metastatic tumor cells. The skeleton belongs to the metastatic sides frequently affected by breast cancer. The prevalence of ESR1 mutation in bone metastasis and the corresponding phenotype are not known. In this study bone metastases from breast cancer $(n=231)$ were analyzed for ESR1 mutation. In 27 patients $(12 \%)$ (median age 73 years, range: 55-82 years) activating mutations of ESR1 were detected. The most frequent mutation was $p$. D538G (53\%), no mutations in exon 4 (K303) or 7 (S463) were found. Lobular breast cancer was present in $52 \%$ of mutated cases $(n=14)$ and in $49 \%$ of all samples $(n=231)$, respectively. Mutated cancers constantly displayed strong estrogen receptor expression. Progesterone receptor was positive in $78 \%$ of the mutated cases $(n=21)$. From 194 estrogen receptor-positive samples, 14\% had ESR1 mutated. Except for one mutated case, no concurrent HER2 overexpression was noted. Metastatic breast cancer with activating mutations of ESR1 had a higher Ki67 labeling index than primary luminal cancers (median $30 \%$, ranging from 5 to $60 \%$ with $85 \%$ of cases revealing $\geq 20 \%$ Ki67-positive cells). From those patients from whom information on endocrine therapy was available $(n=7)$, two had received tamoxifen only, 4 tamoxifen followed by aromatase inhibitors and one patient had been treated with aromatase inhibitors only. We conclude that ESR1 mutation is associated with estrogen receptor expression and high proliferative activity and affects about $14 \%$ of estrogen receptor-positive bone metastases from breast cancer.

Modern Pathology (2018) 31, 56-61; doi:10.1038/modpathol.2017.95; published online 11 August 2017
\end{abstract}

Bone is the predominant, and often the first, site of breast cancer metastasis; $50-70 \%$ of women who experience metastases present with bone metastases during their disease course. ${ }^{1}$ In postmenopausal women with hormone receptor-positive breast cancer, both the disease itself and its therapeutic treatment with anti-estrogenic agents can negatively impact bone, resulting in decreases in bone mineral density and increases in bone loss. ${ }^{1}$ In order to avoid deleterious bone effects, anti-hormonal therapy is only considered when cancer cells express the estrogen receptor $\alpha .^{2}$ More than $75 \%$ of primary breast cancer belong to the luminal subtype and express estrogen receptor $\alpha{ }^{3}$ The growth of the majority of luminal breast cancers is dependent on estrogen and can be efficiently inhibited by antihormonal therapy. Corresponding agents abrogate estrogenic signaling through impeding the

Correspondence: Professor H Kreipe, MD, Institute of Pathology, Hannover Medical School, Carl-Neuberg-Straße 1, Hannover D-30625, Germany.

E-mail: Kreipe.Hans@MH-Hannover.de

Received 4 May 2017; revised 7 June 2017; accepted 18 June 2017; published online 11 August 2017 transcriptional activity of estrogen receptor $\alpha$ or diminishing estrogen synthesis by aromatase inhibition. ${ }^{4}$ A subfraction of luminal cancers can escape anti-hormonal blockade either by primary or by secondary resistance to therapy. ${ }^{4,5}$ The mechanisms of primary resistance remain to be resolved, a promising approach has been made by DNA sequencing of tumors, which were non-responsive to neoadjuvant anti-hormonal therapy. ${ }^{6}$ Secondary or acquired resistance occurs in virtually all patients with estrogen receptor $\alpha$-positive metastatic breast cancer undergoing endocrine therapy, and is attributed to various mechanisms, including loss of expression, ${ }^{7}$ altered activity of coregulators, and cross-talk between the estrogen receptor $\alpha$ and growth factor signaling pathways. ${ }^{5}$ An alternative mechanism of secondary resistance, first described in single cases, ${ }^{8}$ was further unravelled by analysis of metastatic breast cancer showing progression during anti-estrogenic therapy. ${ }^{9}$ Mutations of the estrogen receptor $\alpha$ encoding gene ESR1 were found in hotspot regions in the ligand-binding domain, resulting in ligand-independent, constitutive estrogen receptor $\alpha$ activity. ${ }^{10}$ In this and following studies, ${ }^{11-15}$ prevalence of ESR1 mutation differed 
markedly between patients who were first exposed to aromatase inhibitors during the adjuvant and metastatic settings. Only rarely, and depending on the sensitivity of the method applied, were activating ESR1 mutations detected in primary treatment-naive cancers. ${ }^{10}$ Most of the studies encompassing larger cohorts of patients were conducted on plasma samples. ${ }^{12-15}$ Mutation detection in circulating tumor DNA may miss a considerable proportion of cases ${ }^{16}$ and is difficult to standardize. ${ }^{17}$ Therefore, in this study, tissue samples from biopsies were used for detection of ESR1 mutation as well as correlation with histologic and intrinsic subtype Figure 1.

\section{Materials and Methods}

Bone marrow biopsies were retrieved from the archive of the bone marrow registry in Hannover. In the years 1998-2015, 243 samples with a clinical history of breast cancer and histologically proven metastasis were identified. From these biopsies, 12 were not further analyzed because of low tumor content $(<5 \%)$. The 231 remaining cases had a median age of 69 years (27-89 years). The study population encompassed exclusively women without any male breast cancer patient. All individuals were Caucasians, predominantly from European countries with one Armenian and one Arab. With regard to histological subtype, 118 cases were of no special type (51\%) and $113(49 \%)$ were lobular. Expression of estrogen receptor $\alpha$ and progesterone receptor, as well as HER2 status were determined according to established guidelines using immunohistochemistry and in situ hybridization in case of equivocal HER2 results. ${ }^{18,19}$ E-Cadherin and Ki67 were detected as described. ${ }^{20}$ With regard to receptor expression, 194 cases (83.9\%) belonged to the luminal subtype and 10 cases $(4.3 \%)$ to the HER2 type, respectively (Table 1).

The study was confirmed by the Ethics Committee of Hannover Medical School. ${ }^{19}$

\section{Immunophenotyping}

For immunohistochemistry, $2 \mu \mathrm{m}$ sections of formalin-fixed paraffin-embedded tumor specimens were mounted on poly-L-lysine-coated slides, and deparaffinized and rehydrated conventionally. Immunohistochemical stainings were performed on a Benchmark Ultra (Ventana, U.S.A.) automated stainer using the CC1-mild or CC1-st protocols for target retrieval and the anti-estrogen receptor $\alpha$ (clone SP1, Ventana, undiluted), anti-progesterone receptor $\alpha$ (clone 1E2, Ventana, undiluted), antiHER2 (clone 4B5, Ventana, undiluted), anti-ECadherin (clone MSK 033, Zytomed, Berlin, Germany, 1:100) and anti-Ki-67 (clone SP6,Thermo Scientific, Germany, 1:100) antibodies. HER2 in situ hybridization was done as described. ${ }^{20}$
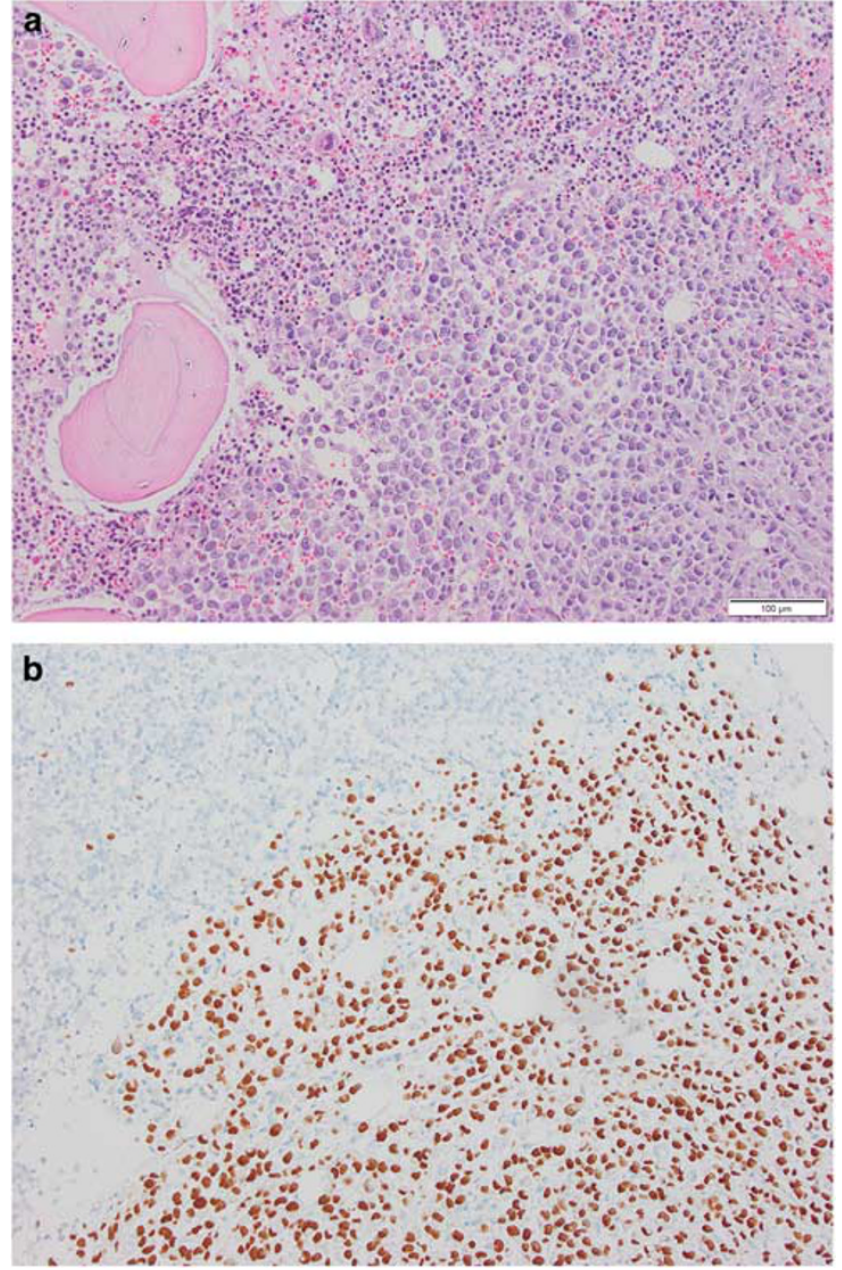

Figure 1 Bone metastasis of lobular breast cancer with ESR1 mutation (a), Hematoxylin-Eosin. In all mutated cases strong estrogen receptor expression was demonstrable (b), immune peroxidase.

Table 1 Age, histologic and intrinsic subtype of ESR1-mutated breast cancer with bone metastases

\begin{tabular}{lll}
\hline & All patients & ESR1 mutated \\
\hline Age (median, range, y= years) & $69 \mathrm{y}, 27 \mathrm{y}-89 \mathrm{y}$ & $73 \mathrm{y}, 55 \mathrm{y}-82 \mathrm{y}$ \\
BC, all types (\%) & $231(100)$ & $27(100)$ \\
BC, no special type (\%) & $118(51.1)$ & $13(48.2)$ \\
BC, lobular (\%) & $113(48.9)$ & $14(51.8)$ \\
ER positive (\%) & $194(84)$ & $27(100)$ \\
PR positive (\%) & $107(46.3)$ & $21(77.8)$ \\
HER2 positive (\%) & $10(4.3)$ & $1(3.7)$ \\
Triple negative (\%) & $33(14.3)$ & $0(0)$ \\
\hline
\end{tabular}

\section{DNA Analysis}

From tumor-bearing paraffin blocks, $10 \mu \mathrm{m}$-thick sections were cut. From each core biopsy, 2-6 sections were taken, depending on its size. Genomic DNA was extracted from formalin-fixed paraffin-embedded specimens with the Maxwell RSC DNA FFPE KIT on a Maxwell ${ }^{\circledR}$ RSC instrument 
Table 2 Genomic location on chromosome 6 and amplicon primer sequences for ESR1 mutation profiling with NGS

\begin{tabular}{|c|c|c|c|c|c|}
\hline & Start & End & $\begin{array}{c}\text { Amplicon } \\
\text { length }\end{array}$ & Forward primer & Reverse primer \\
\hline Exon 4 & 152265409 & 152265540 & 131 bp & 5'-GGTCTGCTGGAGACATGAGAG-3' & 5'-TCACTGAAGGGTCTGGTAGGATC-3' \\
\hline Exon 7 & 152415468 & 152415596 & $128 \mathrm{bp}$ & 5'-CCATGAACACTCTGGGTCTCCTA-3' & 5'-CATCAGGTGGATCAAAGTGTCTGT-3' \\
\hline Exon 8 & 152419900 & 152420028 & $128 \mathrm{bp}$ & 5'-TGGAGCATCTGTACAGCATGAAG-3' & 5'-TGCAAGGAATGCGATGAAGTAGAG-3' \\
\hline
\end{tabular}

Table 3 Pyrosequencing primer used for mutation profiling in exon 5 and for validation of mutations in exon 4, 7, and 8

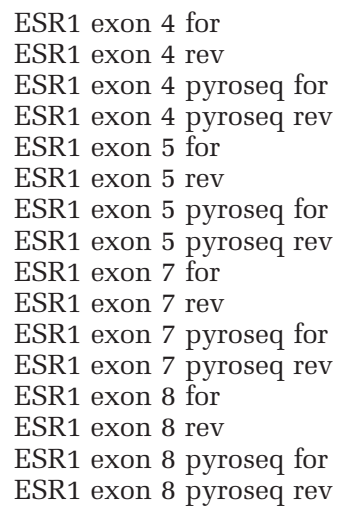

\author{
5'-GGAGACATGAGAGCTGCCAACC-3' \\ 5'-AATAGAGTATCGGGGGCTCAGCA-3' \\ 5'-ATGATCAAACGCTCTAAG-3' \\ 5'-AAGGCCAGGCTGTTC-3' \\ 5'-GTTTTCAGGCTTTGTGGATTTGAC-3' \\ 5'-ACGAGACCAATCATCAGGATCTCT-3' \\ 5'-TCTAGCCAGGCACATT-3' \\ 5'-ATCAGGTCCACCTTCTA-3' \\ 5'-TCTCTGCGCATTCAGGAGTGT-3' \\ 5'-TGCCTTGGCCATCAGGTG-3' \\ 5'-GGAGTGTACACATTTCTG-3' \\ 5'-AGAGACTTCAGGGTGCTGG-3' \\ 5'-TGGAGCATCTGTACAGCATGAAG-3' \\ 5'-GGCGTCCAGCATCTCCAG-3' \\ 5'-AAGAACGTGGTGCCCC-3' \\ 5'-GCATCTCCAGCAGCAG-3'
}

(Promega, Madison, WI, USA) according to the manufacturer's recommendations. DNA quantification was performed using the Qubit 2.0 Fluorometer with dsDNA high sensitivity Assay kit (ThermoFisher Scientific, Dreieich, Germany). Ampliconbased library preparation was performed with a customized NGS mini panel (ESR1 coding regions of exon 4, 7, and 8), consists of short amplicons below 150 bp (Table 2). Next-generation sequencing, data processing, and evaluation were performed as described previously 21

Sequencing of ESR1 exon 5 was performed by pyrosequencing as described. ${ }^{22}$ All mutations in ERS1 detected with NGS were confirmed by pyrosequencing (Table 3).

Digital polymerase chain reaction was performed to evaluate possible ESR1 gene amplification. QuantStudio 3D Digital PCR System, 3D PCR Master Mix v2 and 3D PCR $20 \mathrm{~K}$ Chip Kit v2 were used (ThermoFisher Scientific, Dreieich, Germany). The reaction conditions were as follows: hot start at $96{ }^{\circ} \mathrm{C}$ for $10 \mathrm{~min}$, denaturation at $98^{\circ} \mathrm{C}$ for $30 \mathrm{~s}$, annealing/extension at $62^{\circ} \mathrm{C}$ for $2 \mathrm{~min}$ for a total of 39 cycles, followed by a final extension step at $60^{\circ} \mathrm{C}$ for $2 \mathrm{~min}$. The data analysis was performed with QuantStudio 3D AnalysisSuite ${ }^{\mathrm{TM}}$ Cloud Software version 3.1.2. TaqMan $^{\circledR}$ Copy Number Assays (ESR1 assay ID: Hs00482211_cn; Reference assay: RPPH1, catalog number: 4403326, ThermoFisher Scientific) were used with $30 \mathrm{ng}$ DNA input.

\section{Results}

\section{Frequency and Types of ESR1 Mutations}

Activating mutations of ESR1 were found in 27 patients with bone metastasis from breast cancer. Except for one sample, mutations were exclusively detected in exon 8 with p.D538G being the most frequent type $(n=17$, Table 4$)$, followed by mutations of codon 537 (p.Y537). One patient revealed double mutation with p.D538G and p.E380Q (Table 4). No mutations of exons 4 and 7 were seen. Four patients had received sequential biopsies with up to four further samples and spanning a time period from 1 to 7 years. In three of the patients with follow-up biopsies, the mutation and its allelic burden remained stable over time. One patient with 7 years of follow-up had ESR1 wild type in the first and second biopsy after 3 years. After another 6 years, p.E380Q mutation was detected. In the fourth biopsy after 7 years, a second mutation was demonstrable (p.D538G), which occurred in conjunction with p.E380Q mutation.

\section{ESR1 Gene Amplification}

To evaluate ESR1 gene amplification as a potential second mechanism of resistance towards endocrine therapy copy number variation analysis by digital polymerase chain reaction was performed in a subgroup of samples. From 54 estrogen receptor $\alpha$-positive samples, $53(98 \%)$ samples provided 
Table 4 Types of ESR1 mutations

\begin{tabular}{lccc}
\hline Mutation & $\begin{array}{c}\text { All mutated } \\
\text { cases }\end{array}$ & $\begin{array}{c}\text { No special } \\
\text { type BC }\end{array}$ & Lobular BC \\
\hline p.D538G & 17 & 8 & 9 \\
p.Y537S & 4 & 2 & 2 \\
p.Y537N & 3 & 1 & 2 \\
p.Y537C & 1 & 1 & 0 \\
p.L536H & 2 & 1 & 1 \\
p.E380Q & 1 & 1 & 0 \\
Double mutation & 1 & 1 & 0 \\
p.D538G/p.E380Q & & & \\
\hline
\end{tabular}

evaluable results. In none of these samples ESR1 gene amplification was detectable.

\section{Histologic Types of Metastatic Breast Cancer with ESR1 Mutation}

Lobular and no special type breast cancer were affected by ESR1 mutations in an almost equal frequency. The propensity for lobular breast cancer to metastasize to the bone has been described before, ${ }^{23,24}$ but the high proportion of almost $50 \%$ of all bone metastases by breast cancer, as found in this study (Table 1), had not been expected. No clearcut differences with regard to the share of lobular cancers were found between ESR1-mutated and ESR1-non-mutated cases (Table 4) as well as between estrogen receptor $\alpha$-positive (49\%) or estrogen receptor $\alpha$-negative breast cancer (46\%), making it unlikely that these factors significantly contributed to the over-representation of lobular breast cancer in bone metastases.

Expression of Hormone Receptors, HER2 and Ki67 by Metastatic Breast Cancer with ESR1 Mutation

All ESR1-mutated breast cancers expressed the corresponding receptor and no mutation was found in estrogen receptor $\alpha$-negative cases (Table 1). In 24 patients (89\%), strong estrogen receptor $\alpha$ expression (100\% of cells; Figure 1) occurred, in the remaining 3 cases, estrogen receptor $\alpha$ was positive in $30-80 \%$ of cells. Progesterone receptor expression was present in $78 \%$ of mutated cases $(n=21)$ ranging from 5 to $100 \%$. Among the ESR1-mutated cases, there was one with HER2 overexpression. Metastatic breast cancer with activating mutations of ESR1 displayed a Ki67 labeling index ranging from 5 to $60 \%$. The median labelling index was 30 , and $85 \%$ of cases showed $\geq 20 \%$ Ki67-positive cells.

\section{Endocrine Pretreatment of Metastatic Breast Cancer with ESR1 Mutation}

ESR1 mutation was demonstrable in bone biopsies from patients with first diagnosis of metastatic disease $(n=16)$ as well as in patients with known metastatic disease, and being already under treatment $(n=11)$. In one of the latter patients from whom follow-up biopsies were available, it could be shown that two different ESR1 mutations were acquired during aromatase inhibitor treatment. From seven patients, information on endocrine therapy was available. Two of these patients had been treated exclusively with tamoxifen. Four patients had received tamoxifen followed by aromatase inhibitor, and one patient had been treated with aromatase inhibitor only. In those patients from whom information on endocrine pretreatment could not be retrieved, there is high probability that all of them had received anti-hormonal therapy because the German guidelines require tamoxifen or aromatase inhibitor treatment of every receptor-positive breast cancer irrespective of its grade or stage. ${ }^{25}$ The adherence to the corresponding guide line, specifically to the recommendations on adjuvant endocrine therapy, is high and above $80 \% .{ }^{26}$ Because aromatase inhibitor treatment is recommended in postmenopausal patients and in lobular breast cancer patients ${ }^{25}$ who provide the majority of ESR1-mutated cases in this study, most of the mutated cases have probably been treated with an aromatase inhibitorcontaining regimen.

\section{Discussion}

In this study, which is the largest on bone metastasis of breast cancer so far, $14 \%$ of estrogen receptor $\alpha$ positive cancers harbored an activating ESR1 mutation. A similar proportion has been found in a smaller series encompassing 11 patients. ${ }^{12}$ The estrogen receptor $\alpha$ mutations in this study were clustered in the mutational 'hotspot' surrounding residues 536-538 in the hormone-binding domain, known to result in a constitutive activation of the receptor. ${ }^{2}$ The most frequent ESR1 'hotspot' mutations in metastatic breast cancer are the p.E380Q, p. Y537N, p.Y537S, and p.D538G somatic alterations. ${ }^{2}$ All of these were found in the study population of bone metastases (Table 2). In addition, rarer mutations were encountered in three patients (p.Y537C, p.L536H). Breast cancer of no special type and of lobular type did not differ with regard to distribution of ESR1 mutations (Table 2). One patient showed two types of ESR1 mutations which were sequentially acquired during treatment of metastatic disease. Wang et al. had made similar observations with multiple ESR1 mutations in the same tumor and provided evidence that in these cases, mutations ( $p$. Y537S and p.D538G) were on different alleles, indicating polyclonal disease. ${ }^{12}$ All ESR1-mutated cases in the current study did express estrogen receptor $\alpha$, whereas triple negative cases displayed wild-type ESR1. This is in line with previous findings. ${ }^{13}$

Bone is the preferred metastatic niche for some cancers, including breast cancer, in particular estrogen receptor $\alpha$-positive luminal $\mathrm{BC}$ and it is still 
unclear why such selectivity is seen for bone. ${ }^{2}$ With almost $50 \%$ of cases belonging to the lobular subtype, this study substantiates prior clinical observational studies that lobular BC, exhibits a higher propensity for bone metastasis than breast cancer of no special type..$^{23,24}$ The rate was 3-5-fold of what could have been expected from the prevalence of the lobular subtype among all types of breast cancer (Table 1). Two potential explanations for this over-representation of lobular breast cancer could be envisaged. It may be presumed that higher estrogen receptor $\alpha$ expression in lobular breast cancer might play a role, but a considerably higher share of lobular breast cancer was present in both, the estrogen receptor $\alpha$-positive as well as estrogen receptor $\alpha$-negative subgroup of bone metastasis. Furthermore, it could have been speculated that a higher rate of ESR1 mutation in lobular breast cancer contributes to its over-representation. Obviously, this does not seem to be the case (Table 2). Thus, other factors intrinsic to luminal subtype appear to determine its bone metastatic potential.

There has been some indication that accumulation of ESR1 mutations upon aromatase inhibition might occur especially in bone metastatic tumors, suggesting a selective role of bone microenvironment for such mutations. ${ }^{2}$ But up to now there has been not enough evidence to support a particular role of ESR1 mutation in bone. ${ }^{2}$ Schiavon and colleagues 13 described a high proportion of bone metastasis $(84 \%)$ in their series of 19 ESR1-mutated breast cancer patients. From our study, no convincing evidence can be derived that ESR1 mutation selects for bone metastasis. The frequency of $12 \%$ ESR1mutated cases in all bone metastases and $14 \%$ in the estrogen receptor $\alpha$-positive subgroup, respectively is well in line with other studies which did not focus on metastatic bone disease. ${ }^{12,13,17}$ ESR1 mutations were detected in the plasma of $11 \%$ (18 of 171) of patients with advanced breast cancer, amounting to $14 \%$ of the estrogen receptor $\alpha$-positive cases. ${ }^{13}$ Using cell-free DNA coupled with sensitive digital drop polymerase chain reaction methods, the frequency of ESR1 mutations in metastatic patients is now estimated to be as high as 30 and $50 \%$ in breast cancer. ${ }^{27}$ Although different sensitivities of the methods applied such as droplet ${ }^{12}$ or multiplex polymerase chain reaction ${ }^{13}$ could cause some differences in the detection rate, our data do indicate that the frequency might not be as high as anticipated. ${ }^{27}$ As obvious from one patient in our series who acquired ESR1 mutation during treatment of metastatic disease after being initially negative, the proportion might be higher when aromatase inhibitor treated patients in a metastatic setting are analyzed. ${ }^{13,17}$

Because ESR1 mutations have only rarely been detected in primary tumors, it has been concluded that these mutations appear to be selected by anti-hormonal therapy, in particular aromatase inhibitor treatment. ${ }^{13,27}$ In this study, all patients from whom clinical information was available had received anti-hormonal treatment, the majority with an aromatase inhibitor-containing regimen (5 out of 7 ). In two patients with ESR1 mutation, only tamoxifen had been applied. Using digital drop polymerase chain reaction, Gelsomino et $a .^{28}$ identified mutations at high frequencies ranging from $12 \%$ for p.Y537N, $5 \%$ for p.Y537S, and $2 \%$ for p.D538G in archived primary breast tumors from women treated with adjuvant monotamoxifen therapy. No association of ESR1 mutation with prior tamoxifen exposure was reported by others. ${ }^{13}$

In clinical practice, estrogen receptor $\alpha$ expression is equalled with potential sensitivity to antihormonal therapy. In metastatic disease, endocrine therapy is even considered reasonable regardless of whether the receptor assay had been repeated on the metastasis once the primary tumor had yielded a positive hormone receptor assay. ${ }^{29}$ From first clinical trials, there is growing evidence for a worse outcome for ESR1-mutated cases when further treated with aromatase inhibitor instead of fulvestrant. ${ }^{15}$ Consequently, reporting receptor positivity of metastatic disease might not be sufficient anymore and information on the ESR1 mutation status should be included in order to guide adequate therapy. Preliminary evidence suggests that some of the ESR1 mutations are sensitive to fulvestrant, and/or everolimus, or palbociclib treatment, thus these agents promise to be potentially useful in metastatic ESR1 mutation-positive BC patients. $^{2,15}$

\section{Acknowledgments}

This study was supported by a grant from the Deutsche Krebshilfe to HK (Grant Number 1097154)

\section{Disclosure/conflict of interest}

The authors declare no conflict of interest.

\section{References}

1 Yardley DA. Pharmacologic management of bone-related complications and bone metastases in postmenopausal women with hormone receptorpositive breast cancer. Breast Cancer 2016;8:73-82.

2 Bado I, Gugala Z, Fuqua SA, et al. Estrogen receptors in breast and bone: from virtue of remodeling to vileness of metastasis. Oncogene 2017. e-pub ahead of print 3 April 2017.

3 Howlader N, Altekruse SF, Li CI, et al. US incidence of breast cancer subtypes defined by joint hormone receptor and HER2 status. J Natl Cancer Inst 2014;106: $1-8$.

4 Patani N, Martin LA. Understanding response and resistance to oestrogen deprivation in ER-positive breast cancer. Mol Cell Endocrinol 2014;382:683-694. 
5 Ma CX, Reinert T, Chmielewska I, et al. Mechanisms of aromatase inhibitor resistance. Nat Rev Cancer 2015;15: 261-275.

6 Gellert P, Segal CV, Gao Q, et al. Impact of mutational profiles on response of primary oestrogen receptorpositive breast cancers to oestrogen deprivation. Nat Commun 2016;7:13294.

7 Gaedcke J, Traub F, Milde S, et al. Predominance of the basal type and HER-2/neu type in brain metastasis from breast cancer. Mod Pathol 2007;20:864-870.

8 Zhang QX, Borg A, Wolf DM, et al. An estrogen receptor mutant with strong hormone-independent activity from a metastatic breast cancer. Cancer Res 1997;57:1244-1249.

9 Merenbakh-Lamin K, Ben-Baruch N, Yeheskel A, et al. D538G mutation in estrogen receptor- $\alpha$ : a novel mechanism for acquired endocrine resistance in breast cancer. Cancer Res 2013;73:6856-6864.

10 Toy W, Shen Y, Won H, et al. ESR1 ligand-binding domain mutations in hormone-resistant breast cancer. Nat Genet 2013;45:1439-1445.

11 Robinson DR, Wu YM, Vats P, et al. Activating ESR1 mutations in hormone-resistant metastatic breast cancer. Nat Genet 2013;45:1446-1451.

12 Wang P, Bahreini A, Gyanchandani R, et al. Sensitive detection of mono- and polyclonal ESR1 mutations in primary tumors, metastatic lesions, and cell-free DNA of breast cancer patients. Clin Cancer Res 2016;22: 1130-1137.

13 Schiavon G, Hrebien S, Garcia-Murillas I, et al. Analysis of ESR1 mutation in circulating tumor DNA demonstrates evolution during therapy for metastatic breast cancer. Sci Transl Med 2015;7:313ra182.

14 Sefrioui D, Perdrix A, Sarafan-Vasseur N, et al. Short report: Monitoring ESR1 mutations by circulating tumor DNA in aromatase inhibitor resistant metastatic breast cancer. Int J Cancer 2015;137:2513-2519.

15 Fribbens C, O'Leary B, Kilburn L, et al. Plasma ESR1 mutations and the treatment of estrogen receptorpositive advanced breast cancer. J Clin Oncol 2016;34: 2961-2968.

16 Sundaresan TK, Sequist LV, Heymach JV, et al. Detection of T790M, the acquired resistance EGFR mutation, by tumor biopsy versus noninvasive blood-based analyses. Clin Cancer Res 2016;22:1103-1110.

17 Jeselsohn R, De Angelis C, Brown M, et al. The Evolving Role of the estrogen receptor mutations in endocrine therapy-resistant breast cancer. Curr Oncol Rep 2017;19:35.

18 Hammond ME, Hayes DF, Dowsett M, et al. American Society of Clinical Oncology/College of American
Pathologists guideline recommendations for immunohistochemical testing of estrogen and progesterone receptors in breast cancer (unabridged version). Arch Pathol Lab Med 2010;134:e48-e72.

19 Wolff AC, Hammond ME, Hicks DG, et al. Recommendations for human epidermal growth factor receptor 2 testing in breast cancer: American Society of Clinical Oncology/College of American Pathologists clinical practice guideline update. J Clin Oncol. 2013;31: 3997-4013.

20 Gluz O, Nitz UA, Christgen M, et al. West German Study Group Phase III PlanB Trial: first prospective outcome data for the 21-gene recurrence score assay and concordance of prognostic markers by central and local pathology assessment. J Clin Oncol 2016;34: 2341-2349.

21 Bartels S, Schipper E, Hasemeier B, et al. Routine clinical mutation profiling using next generation sequencing and a customized gene panel improves diagnostic precision in myeloid neoplasms. Oncotarget 2016;7:30084-30093.

22 Bartels S, Lehmann U. Analysis of mutational hotspots in routinely processed bone marrow trephines by Pyrosequencing. Methods Mol Biol. 2015;1315: 103-114.

23 Jain S, Fisher C, Smith P, et al. Patterns of metastatic breast cancer in relation to histological type. Eur J Cancer 1993;29 A:2155-2157.

24 Borst MJ, Ingold JA. Metastatic patterns of invasive lobular versus invasive ductal carcinoma of the breast. Surgery 1993;114:637-641.

25 Thomssen C, Scharl A, Harbeck N. AGO recommendations for diagnosis and treatment of patients with primary and metastatic breast cancer. update 2011. Breast Care 2011;6:299-313.

26 Wolters R, Wischhusen J, Stüber T, et al. Guidelines are advantageous, though not essential for improved survival among breast cancer patients. Breast Cancer Res Treat 2015;152:357-366.

27 Takeshita T, Yamamoto Y, Yamamoto-Ibusuki M, et al. Clinical significance of monitoring ESR1 mutations in circulating cell-free DNA in estrogen receptor positive breast cancer patients. Oncotarget 2016;7:32504-32518.

28 Gelsomino L, Gu G, Rechoum Y, et al. ESR1 mutations affect anti-proliferative responses to tamoxifen through enhanced cross-talk with IGF signaling. Breast Cancer Res Treat 2016;157:253-265.

29 Carlson RW, Allred DC, Anderson BO, et al. Metastatic breast cancer, version 1.2012: featured updates to the NCCN guidelines. J Natl Compr Canc Netw 2012;10: 821-829. 